\title{
Feed Barley Genotypes Evaluated for Adaptability under Multi Environment Field Trials of North Eastern Plains Zone of the Country
}

\author{
Ajay Verma*, R. P. S. Verma, J. Singh, L. Kumar and G. P. Singh \\ ICAR-Indian Institute of Wheat and Barley Research, Karnal Haryana, India \\ *Corresponding author
}

\section{A B S T R A C T}

Keywords

AMMI, ASV, ASV1, HMGV, GAI, HMPRVG, Biplots

Article Info

\section{Accepted:}

12 April 2021

Available Online:

10 May 2021
Highly significant effects of the environment (E), genotypes $(\mathrm{G})$, and $\mathrm{GxE}$ interaction had been observed by AMMI analysis. GxE interaction accounted for $45.8 \%$ whereas Environment explained $27.4 \%$ of treatment variations in yield during first year. Ranking of genotype as per IPCA-1 were RD2969, K508. While IPCA-2, selected K508, HUB113 genotypes. Values of ASV1 selected RD2969, K508 and ASV identified K508, HUB113 barley genotypes. Adaptability measures Harmonic Mean of Relative Performance of Genotypic Values (HMPRVG) and Relative Performance of Genotypic Values (RPGV) identified DWRB137, HUB113 as the genotypes of performance among the locations. Biplot graphical analysis exhibited cluster of adaptability measures PRVG, HMPRVG along with mean, GM, HM. During 2019-20 cropping season Environment effects accounted $37.1 \%$ whereas GxE interaction contributed for $29.2 \%$ of treatment variations in yield. IPCA-1 scores, desired ranking of genotype was KB1815, DWRB213, RD3021. While IPCA-2 pointed towards RD3019, NDB1748, KB1815 as genotypes of choice. Analytic measures ASV and ASV1 selected KB1815, DWRB213, RD3021 barley genotypes. HMRPGV along with PRVG settled for DWRB213, Lakhan, KB1832. Measures IPC2, IPC3, IPC6 clustered with adaptability measures PRVG, HMPRVG, mean, GM, HM in separate cluster and observed in different quadrant of biplot analysis.

\section{Introduction}

Most cosmopolitan crop, Barley (Hordeum vulgare $L$.) grown over the wide range of environmental conditions of the country (Kharub et al., 2017; Bocianowsk et al., 2019). Popularly famous, as "poor man's crop" owing to low requirements of input along with better adaptability to harsh conditions (Kendel et al., 2019). Feed barley is mainly cultivated as a fodder for animal consumption as enriched with nutrients and possess medicinal properties. Traditionally the crop cultivated for grains as crop for human consumption as well feed for animals (Karkee et al., 2020). On yearly basis number of multilocation trials under coordinated system carried out for GxE interaction analysis (Agahi et al., 2020). Breeders select or identify genotypes with stable yield along with 
broad or narrow adaptation bahaviour of the genotypes (Bocianowsk et al., 2019). Number of adaptability measures based on AMMI stability had observed in literature (Tekdal \& Kendal, 2018; Ajay et al., 2019). Analytic measure of adaptability as the harmonic means of the relative performance of the predicted genotypic values (MHPRVG) utilized productivity, stability, and adaptability simultaneously of genotypes (Resende \& Durate 2007). Comparative performance of AMMI based measures had been studied with relatively new adaptability measures for feed barley genotypes evaluated under North Eastern Plains Zone of the country in recent past.

\section{Materials and Methods}

States of the country Bihar, eastern Uttar Pradesh, Jharkhand, Assam and plains of West Bengal comprises the North Eastern Plains Zone of India. This zone has potential to increase the total production and importance of this zone has been highlighted to ensure food security of the country. Total of six promising genotypes evaluated at five major locations and fifteen genotypes at eight locations of the zone during cropping seasons of 2018-19 and 2019-20 respectively. Field trials were conducted at research centers in randomized complete block designs with three replications. Recommended agronomic practices were followed to harvest good yield. Details of locations and genotype parentage were reflected in tables $1 \& 2$ for ready reference.

AMMI analysis was performed using AMMISOFT version 1.0, available at https://scs.cals.cornell.edu/people/ hughgauch/and SAS software version 9.3. Simple and effective measure for adaptability is calculated as the relative performance of genetic values (PRVG) across environments and MHVG (Harmonic mean of Genetic
Values), based on the harmonic mean of the genotypic values across in different environments. Lower the standard deviation of genotypic performance across environments, the greater is the harmonic mean of its genotypic values.

\section{Results and Discussion}

\section{AMMI analysis of barley genotypes}

\section{First year of study 2018-19}

AMMI based measures evaluate the adaptability performance after reduction of the noise from the GxE interaction effects (Gauch, 2013). Highly significant effects of the environment $(\mathrm{E})$, genotypes $(\mathrm{G})$, and $\mathrm{GxE}$ interaction had been observed by AMMI analysis (Table 3). Analysis observed the greater contribution of environments, GxE interactions, and genotypes to the total sum of squares (SS) as compared to the residual effects (Kamila et al., 2016). Environment explained about significantly $27.4 \%$ of the total sum of squares due to treatments indicating that diverse environments caused most of the variations in genotypes yield. Genotypes explained only $13.5 \%$ of a total sum of squares, whereas GxE interaction accounted for $45.8 \%$ of treatment variations in yield. Further bifurcation of $\mathrm{GxE}$ interaction observed the significant three multiplicative terms explained $99 \%$ of interaction sum of squares and the remaining $1.0 \%$ was the residual / noise, which was not interpretable and discarded (Oyekunle et al., 2017).

\section{Second year 2019-20}

Analysis observed the greater contribution of environments, GxE interactions, and genotypes to the total sum of squares (SS) as compared to the residual effects. Environment explained about significantly $37.1 \%$, GxE interaction accounted for 29.2 whereas 
Genotypes explained only $10.5 \% \%$ of the total sum of squares due to treatments. Partitioning of GxE interaction revealed that only first three out of six multiplicative terms were significant and explained of interaction sum of squares.

\section{Ranking of genotypes as per descriptive measures}

\section{First year of study 2018-19}

An average yield of genotypes selected DWRB137, HUB113 genotypes (Table 5). This method is simple, but not fully exploiting all information contained in the dataset. Geometric mean is used to evaluate the adaptability of genotypes. Geometric mean observed DWRB137, HUB113 were topranked genotypes. Harmonic mean of genetic values (HMGV) yield expressed higher values for DWRB137, HUB113genotypes.

Consistent yield performance judged by lower values of Coefficient of Variation and genotypes DWRB137, RD 2552would be suitable for considered locations of this zone of the country. Minimum values of standard deviation of yield values selected DWRB137, RD 2552, barley genotypes. Analytic measures PRVG, MHVG, and MHPRVG, had showed consensus for classification of genotypes as per raking of genotypes vis-à-vis analytic measures (Table 4). Presence of significant cross over interactions has been validated by differences among ranks of genotypes vis-à-vis locations of the zone.

\section{Second year 2019-20}

An average yield of genotypes selected Lakhan, DWRB213, KB1832 genotypes (Table 9). Geometric mean observed Lakhan, DWRB213, KB1832, were with top-rank. Harmonic mean of genetic values (HMGV) expressed higher values for Lakhan, DWRB213, HUB69 genotypes.

Consistent yield performance of Lakhan, DWRB213, HUB270 judged by lower values of Coefficient of Variation. Minimum values of standard deviation of yield values selected Lakhan, HUB270, NDB1748 barley genotypes. Analytic measures PRVG, MHVG, and MHPRVG, had showed consensus for classification of genotypes as per raking of genotypes vis-à-vis analytic measures (Table 6). Presence of significant cross over interactions has been validated by differences among ranks of genotypes vis-à-vis locations of the zone.

\section{Adaptability behaviour of genotypes}

\section{First year of study 2018-19}

The IPCA scores of a genotype in AMMI analysis indicate the stability or adaptation over environments. The greater the IPCA scores, either negative or positive (as it is a relative value), the more specifically adapted is the genotype to certain environments. The more the IPCA scores approximate zero, the more stable or adapted the genotypes are over the entire environments sampled (Ajay et al., 2019). Kendal and Tekdal, 2016 stated that genotypes having PC1 scores > 0 were recognized as high-yielding and those having PC1 scores $<0$ were regarded as low-yielding. The IPCA scores of genotypes in the AMMI analysis are an indication of stability or adaptability over environments. The ranking of genotype as per absolute IPCA-1 scores were RD2969, K508(Table 4). While for IPCA-2, genotypes K508, HUB113would be of choice. Values of IPCA-3 favored RD 2552, K1055barley genotypes. Analytic measures of adaptability ASV and ASV1consider two significant IPCAs of the AMMI analysis for adaptability behaviour. 
Table.1

\begin{tabular}{|c|c|c|}
\hline $\begin{array}{l}\text { Mohamadi \& Amri } \\
2008\end{array}$ & $\begin{array}{l}\text { Geometric Adaptability } \\
\text { Index }\end{array}$ & $\mathbf{G A I}=\sqrt[n]{\prod_{\mathrm{k}=1}^{\mathrm{n}} \overline{\mathrm{X}}_{\mathrm{k}}}$ \\
\hline Purchase1997 & AMMI stability value & $\mathrm{ASV}=\left[\left(\frac{\text { SISIFE 1 }}{\mathrm{SIFE2}} P(I)^{2}+(P C 2)^{2}\right]^{1 / 2}\right.$ \\
\hline Zali et al2012 & AMMI stability value & ASV1 $=\left[\frac{\operatorname{SSIPC1} 1}{\operatorname{SIPC2} 2}(P C I)^{2}+(P C 2)^{2}\right]^{1 / 2}$ \\
\hline Resende 2004 & $\begin{array}{l}\text { Harmonic mean of Genetic } \\
\text { Values }\end{array}$ & $\mathrm{MHVG}_{\mathrm{i}}=$ Number of environments $/ \sum_{i=1}^{k} \frac{1}{x_{i}}$ \\
\hline $\begin{array}{l}\text { Resende\&Durate } \\
2007\end{array}$ & $\begin{array}{l}\text { Relative performance of } \\
\text { genotypic values across } \\
\text { environments }\end{array}$ & $\mathrm{PRVG}_{\mathrm{ij}}=\mathrm{VG}_{\mathrm{ij}} / \mathrm{VG}_{\mathrm{i}}$ \\
\hline $\begin{array}{l}\text { Resende\&Durate } \\
2007\end{array}$ & $\begin{array}{l}\text { Harmonic mean of Relative } \\
\text { performance of genotypic } \\
\text { values }\end{array}$ & MHPRVG $_{\mathrm{i} .}=$ Number of environments $/ \sum_{j=1}^{k} \frac{1}{P R G_{i j}}$ \\
\hline
\end{tabular}

Table.2 Parentage details of barley genotypes and environmental conditions (2018-19)

\begin{tabular}{|c|c|c|c|c|c|c|c|}
\hline Code & Genotype & Parentage & Code & Location & Latitude & Longitude & Altitude \\
\hline G1 & RD 2552 & RD2035/DL472 & E1 & Varanasi & $25^{\circ} 19^{\prime} \mathrm{N}$ & $82^{\circ} 59^{\prime} \mathrm{E}$ & 81 \\
\hline G2 & K1055 & & E2 & Faizabad & $26^{\circ} 46^{\prime} \mathrm{N}$ & $82^{\circ} 9^{\prime} \mathrm{E}$ & 97 \\
\hline G3 & HUB113 & KARAN280/C138 & E3 & Kanpur & $26^{\circ} 26^{\prime} \mathrm{N}$ & $80^{\circ} 19^{\prime} \mathrm{E}$ & 126 \\
\hline G4 & RD2969 & RD2552/RD2503//RD 2715 & E4 & Ranchi & $23^{\circ} 20^{\prime} \mathrm{N}$ & $85^{\circ} 18^{\prime} \mathrm{E}$ & 651 \\
\hline G5 & DWRB137 & DWR28/DWRUB64 & E5 & Sabour & $25^{\circ} 23^{\prime} \mathrm{N}$ & $87^{\circ} 04^{\prime} \mathrm{E}$ & 46 \\
\hline G6 & K508 & K394/K141 & & & & & \\
\hline
\end{tabular}

Table.3 Parentage details of barley genotypes and environmental conditions (2019-20)

\begin{tabular}{|c|c|c|c|c|c|c|c|}
\hline Code & Genotype & Parentage & Code & Location & Latitude & Longitude & Altitude \\
\hline G1 & NDB1748 & CEV96060/MSEL//CANELA ACBSSO & E1 & Kanpur & $26^{\circ} 26^{\prime} \mathrm{N}$ & $80^{\circ} 19^{\prime} \mathrm{E}$ & 126 \\
\hline G2 & KB1830 & RD 2784/Jyoti & E2 & Saini & $28^{\circ} 12^{\prime} \mathrm{N}$ & $75^{\circ} 40^{\prime} \mathrm{E}$ & \\
\hline G3 & RD3020 & RD 2035/ RD 2624//RD 2715 & E3 & Varanasi & $25^{\circ} 19^{\prime} \mathrm{N}$ & $82^{\circ} 59^{\prime} \mathrm{E}$ & 81 \\
\hline G4 & $\begin{array}{c}\text { DWRB21 } \\
3\end{array}$ & CONCHITA/DWRUB64 & E4 & Faizabad & $\begin{array}{c}26^{\circ} 46^{\prime} \\
\mathrm{N}\end{array}$ & $82^{\circ} 9^{\prime} \mathrm{E}$ & 97 \\
\hline G5 & Lakhan & K12/IB226 & E5 & Chianki & $23^{\circ} 45^{\prime} \mathrm{N}$ & $85^{\circ} 30^{\prime} \mathrm{E}$ & 215 \\
\hline G6 & RD3022 & RD 2607 / RD 2651 & E6 & Ranchi & $23^{\circ} 20^{\prime} \mathrm{N}$ & $85^{\circ} 18^{\prime} \mathrm{E}$ & 651 \\
\hline G7 & HUB269 & 31st INBON-04 / RD 2552 & E7 & Pusa & $28^{\circ} 38^{\prime} \mathrm{N}$ & $77^{\circ} 09^{\prime} \mathrm{E}$ & 52 \\
\hline G8 & PL925 & VJM315/BH919 & E8 & Sabour & $25^{\circ} 23^{\prime} \mathrm{N}$ & $87^{\circ} 04^{\prime} \mathrm{E}$ & 46 \\
\hline G9 & HUB270 & RD-2618 /RD-2660 & & & & & \\
\hline G10 & KB1815 & $\begin{array}{l}\text { Ghinneri(smooth_awns)/6/JLB70- } \\
\text { 01/5/DeirAlla106//DL70/Pyo/3/RM1508 } \\
\text { /4/Arizona5908/Aths//Avt/Attiki/3/Ager }\end{array}$ & & & & & \\
\hline G11 & RD3019 & RD 2715 / RD 2552 & & & & & \\
\hline G12 & K 603 & K257/C138 & & & & & \\
\hline G13 & PL918 & $\begin{array}{l}\text { VMorales/6/LEGACY//PENCO/CHEVRON- } \\
\text { BAR/7/LIGNEE527/GERBEL/3/BOYB* } \\
\text { 2/ SURB//CI12225.2D/4/GLORIA- } \\
\text { BAR/COME }\end{array}$ & & & & & \\
\hline G14 & KB1832 & K 603 x RD 2715 & & & & & \\
\hline G15 & RD3021 & DWR 64 / RD 2503 & & & & & \\
\hline
\end{tabular}


Table.4 Multi environment trails analysis by AMMI of feed barley genotypes (2018-19)

\begin{tabular}{|c|c|c|c|c|}
\hline Source & Degree of freedom & Mean Sum of Squares & Significance level & \% contributions of factors \\
\hline Treatments & 29 & 201.84 & $.0000000 * * *$ & 86.80 \\
\hline Genotypes (G) & 5 & 181.74 & $.0000000 * * *$ & 13.48 \\
\hline Environments (E) & 4 & 462.74 & $.0000000 * * *$ & 27.45 \\
\hline Interactions (GxE) & 20 & 154.68 & $.0000000 * * *$ & $.0000000 * * *$ \\
\hline IPC1 & 8 & 218.29 & $.0000000 * * *$ & \\
\hline IPC2 & 6 & 126.16 & $.0000000 * * *$ & \\
\hline IPC3 & 4 & 135.42 & $.0496326 *$ & \\
\hline Residual & 2 & 24.35 & & \\
\hline Error & 90 & 9.89 & & \\
\hline Total & 119 & 56.67 & & \\
\hline
\end{tabular}

Table.5 Ranking of feed barley genotypes as per descriptive measures (2018-19)

\begin{tabular}{|c|c|c|c|c|c|c|c|c|c|c|c|c|c|c|c|}
\hline Genotype & Varanasi & Faizabad & Kanpur & Ranchi & Sabour & MEAN & $\mathbf{R}_{\mathrm{k}}$ & GM & $\mathbf{R}_{\mathbf{k}}$ & HM & $\mathbf{R}_{\mathbf{k}}$ & CV & $\mathbf{R}_{\mathbf{k}}$ & Sdev & $\mathbf{R}_{\mathbf{k}}$ \\
\hline RD 2552 & 28.25 & 37.15 & 37.41 & 41.84 & 32.95 & 35.52 & 5 & 35.21 & 5 & 34.89 & 5 & 0.1447 & 2 & 5.14 & 2 \\
\hline K1055 & 30.09 & 28.26 & 2.17 & 24.34 & 35.01 & 33.97 & 6 & 32.77 & 6 & 31.76 & 6 & 0.3200 & 6 & 10.87 & 6 \\
\hline HUB113 & 33.82 & 38.96 & 53.62 & 1.38 & 4.16 & 40.39 & 2 & 39.80 & 2 & 39.27 & 2 & & 4 & 8.06 & 5 \\
\hline RD2969 & 24.99 & & & & & 36.64 & 4 & 35.90 & 4 & 35.11 & 4 & 0.2135 & 5 & 7.82 & 4 \\
\hline DWRB137 & 44.14 & 39.81 & 38.59 & 43.34 & 43.05 & 41.78 & 1 & 41.73 & 1 & 41.67 & 1 & 0.0582 & 1 & 2.43 & 1 \\
\hline K508 & 36.76 & 46.21 & 42.39 & 39.01 & 31.22 & 39.11 & 3 & 38.78 & 3 & 38.43 & 3 & 0.1452 & 3 & 5.68 & 3 \\
\hline
\end{tabular}


Table.6 Adaptability measures of feed barley genotypes evaluated under MET (2018-19)

\begin{tabular}{|c|c|c|c|c|c|c|c|c|c|c|c|}
\hline Genotype & IPC1 & IPC2 & IPC3 & ASV1 & R ASV1 $_{\text {ASV }}$ & R $_{\text {ASV }}$ & PRVG & RRVG $_{\text {PRV }}$ & HMPRVG & R \\
\hline HMPRVG \\
\hline RD 2552 & 1.529 & 1.002 & -0.098 & 3.67 & 4 & 2.53 & 4 & 0.9379 & 5 & 0.9311 \\
\hline KU1055 & -3.511 & -1.191 & -0.504 & 8.19 & 6 & 5.46 & 6 & 0.8891 & 6 & 0.8555 \\
\hline RD2969 & -1.176 & 0.885 & 1.048 & 2.85 & 3 & 1.99 & 2 & 1.0584 & 2 & 1.0537 \\
\hline DWRB137 & 1.865 & -2.139 & -1.645 & 4.81 & 5 & 3.55 & 5 & 1.1183 & 1 & 1 \\
\hline K508 & 1.161 & -0.849 & 2.450 & 2.81 & 2 & 1.96 & 1 & 1.0359 & 3 & 1.0984 \\
\hline
\end{tabular}

Table.7 Loadings of adaptability measures as per Principal Components (2018-19)

\begin{tabular}{|c|c|c|}
\hline Component & PC1 & PC2 \\
\hline IPC1 & 0.2626 & 0.1279 \\
\hline IPC2 & -0.0683 & 0.4488 \\
\hline IPC3 & 0.0382 & 0.2540 \\
\hline ASV1 & -0.1916 & -0.4106 \\
\hline ASV & -0.1936 & -0.4209 \\
\hline Varanasi & 0.2299 & -0.3324 \\
\hline Faizabad & 0.2677 & 0.1490 \\
\hline Kanpur & -0.1686 & -0.0447 \\
\hline Ranchi & 0.2569 & 0.2318 \\
\hline Sabour & 0.1138 & -0.3683 \\
\hline Average & 0.2912 & -0.1005 \\
\hline CV & -0.3003 & 0.0246 \\
\hline Sdev & -0.2818 & 0.0575 \\
\hline GM & 0.3011 & -0.0882 \\
\hline HM & 0.3060 & -0.0855 \\
\hline PRVG & 0.2972 & -0.1197 \\
\hline HMPRVG & 0.3030 & -0.0670 \\
\hline variation & 57.35 & 20.39 \\
\hline & & \\
\hline
\end{tabular}


Table.8 Multi environment trails analysis by AMMI of barley genotypes (2019-20)

\begin{tabular}{|c|c|c|c|c|}
\hline Source & Degree of Freedom & Mean Sum of Squares & Significance level & \% contributions of factors \\
\hline Treatments & 119 & 204.15 & $7 * .87$ \\
\hline Genotypes (G) & 14 & 237.83 & $* * *$ \\
\hline Environments (E) & 7 & 1675.30 & $* 34$ \\
\hline Interactions (GxE) & 98 & 94.26 & $* * .11$ \\
\hline IPC1 & 20 & 252.63 & $* *$ \\
\hline IPC2 & 18 & 80.55 & $* *$ \\
\hline IPC3 & 16 & 79.05 & $* *$ \\
\hline IPC4 & 14 & 55.16 & \\
\hline IPC5 & 12 & 27.75 & \\
\hline IPC6 & 10 & 24.29 & \\
\hline Residual & 8 & 15.21 & \\
\hline Trror & 240 & 30.45 & \\
\hline
\end{tabular}

Table.9 Ranking of barley genotypes as per descriptive measures (2019-20)

\begin{tabular}{|c|c|c|c|c|c|c|c|c|c|c|c|c|c|c|c|c|c|c|}
\hline Genotype & Kanpur & Saini & Varanasi & Faizabad & Chianki & Ranchi & Pusa & Sabour & Mean & $\mathbf{R}_{\mathbf{k}}$ & GM & $\mathbf{R}_{\mathbf{k}}$ & HM & $\mathbf{R}_{\mathbf{k}}$ & CV & $\mathbf{R}_{\mathbf{k}}$ & Sdev & $\mathbf{R}_{\mathbf{k}}$ \\
\hline NDB1748 & 38.05 & 18.84 & 30.09 & 28.99 & 27.13 & 36.96 & 28.26 & 19.68 & 28.50 & 9 & 27.72 & 8 & 26.915 & 8 & 0.2440 & 5 & 6.95 & 3 \\
\hline KB1830 & 40.58 & 31.52 & 21.07 & 28.69 & 45.00 & 48.91 & 27.18 & 23.08 & 33.25 & 4 & 31.88 & 5 & 30.593 & 5 & 0.3112 & 9 & 10.35 & 11 \\
\hline RD3020 & 51.18 & 34.42 & 30.00 & 27.72 & 38.23 & 31.52 & 25.73 & 20.39 & 32.40 & 6 & 31.32 & 6 & 30.327 & 6 & 0.2876 & 7 & 9.32 & 8 \\
\hline DWRB213 & 50.00 & 33.52 & 29.78 & 28.87 & 32.89 & 36.96 & 28.26 & 31.06 & 33.92 & 2 & 33.37 & 2 & 32.907 & 2 & 0.2091 & 2 & 7.09 & 4 \\
\hline Lakhan & 35.96 & 25.36 & 41.07 & 29.59 & 41.29 & 35.87 & 30.80 & 31.58 & 33.94 & 1 & 33.53 & 1 & 33.109 & 1 & 0.1654 & 1 & 5.61 & 1 \\
\hline RD3022 & 45.02 & 38.04 & 19.42 & 28.08 & 25.64 & 34.24 & 14.49 & 24.37 & 28.66 & 8 & 27.08 & 10 & 25.489 & 10 & 0.3491 & 10 & 10.00 & 9 \\
\hline HUB69 & 35.14 & 26.09 & 33.35 & 29.77 & 30.38 & 48.37 & 32.43 & 24.78 & 32.54 & 5 & 31.91 & 4 & 31.354 & 3 & 0.2241 & 4 & 7.29 & 5 \\
\hline PL925 & 38.77 & 19.93 & 11.29 & 24.16 & 21.61 & 30.43 & 22.10 & 18.83 & 23.39 & 15 & 22.14 & 13 & 20.862 & 11 & 0.3507 & 11 & 8.20 & 7 \\
\hline HUB270 & 24.46 & 21.74 & 36.25 & 26.87 & 34.92 & 25.54 & 34.06 & 20.38 & 28.03 & 10 & 27.43 & 9 & 26.854 & 9 & 0.2214 & 3 & 6.21 & 2 \\
\hline KB1815 & 40.13 & 33.33 & 6.79 & 27.78 & 36.57 & 36.41 & 12.32 & 19.92 & 26.65 & 11 & 23.15 & 11 & 18.847 & 13 & 0.4631 & 13 & 12.34 & 13 \\
\hline RD3019 & 42.21 & 30.07 & 23.10 & 22.95 & 30.12 & 32.61 & 16.30 & 7.55 & 25.61 & 12 & 23.11 & 12 & 19.926 & 12 & 0.4150 & 12 & 10.63 & 12 \\
\hline K 603 & 26.81 & 18.48 & 43.71 & 27.78 & 28.74 & 34.78 & 23.92 & 28.70 & 29.11 & 7 & 28.31 & 7 & 27.538 & 7 & 0.2572 & 6 & 7.49 & 6 \\
\hline PL918 & 52.08 & 35.33 & 9.61 & 28.02 & 19.27 & 31.96 & 13.77 & 9.17 & 24.90 & 14 & 21.05 & 15 & 17.691 & 15 & 0.5965 & 15 & 14.85 & 15 \\
\hline KB1832 & 51.63 & 32.61 & 22.45 & 29.89 & 32.93 & 46.20 & 30.07 & 23.31 & 33.64 & 3 & 32.37 & 3 & 31.225 & 4 & 0.3060 & 8 & 10.29 & 10 \\
\hline RD3021 & 41.30 & 44.20 & 15.15 & 27.72 & 20.30 & 29.89 & 12.50 & 8.36 & 24.93 & 13 & 21.67 & 14 & 18.548 & 14 & 0.5292 & 14 & 13.19 & 14 \\
\hline
\end{tabular}


Table.10 Adaptability measures of barley genotypes evaluated under MET (2019-20)

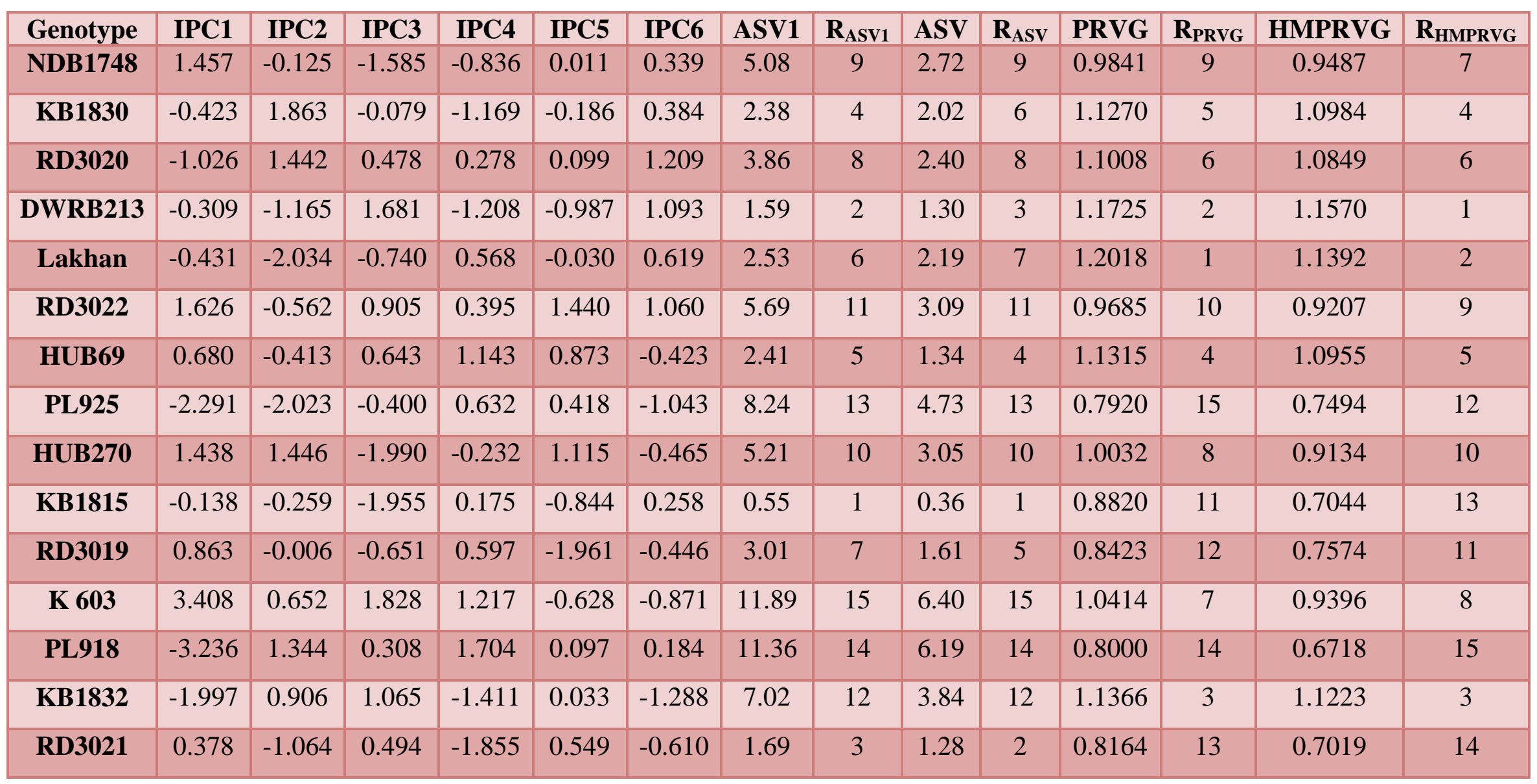


Table.11 Loadings of adaptability measures as per Principal Components (2019-20)

\begin{tabular}{|c|c|c|}
\hline Component & PC1 & PC2 \\
\hline IPC1 & -0.0829 & -0.2306 \\
\hline IPC2 & -0.0117 & 0.0393 \\
\hline IPC3 & -0.0654 & 0.0965 \\
\hline IPC4 & 0.0636 & -0.2449 \\
\hline IPC5 & -0.0247 & -0.0673 \\
\hline IPC6 & -0.0854 & 0.2336 \\
\hline ASV1 & 0.0824 & -0.2879 \\
\hline ASV & 0.0671 & -0.2812 \\
\hline Kanpur & 0.0584 & 0.3973 \\
\hline Saini & 0.1043 & 0.4279 \\
\hline Varanasi & -0.2454 & -0.2399 \\
\hline Faizabad & -0.1959 & 0.1738 \\
\hline Chianki & -0.2239 & 0.1219 \\
\hline Ranchi & -0.1670 & 0.1957 \\
\hline Pusa & -0.2724 & -0.1400 \\
\hline Sabour & -0.2740 & -0.0596 \\
\hline Mean & -0.2976 & 0.1661 \\
\hline CV & 0.2929 & 0.1651 \\
\hline Sdev & 0.2441 & 0.2705 \\
\hline PRVG & -0.3131 & 0.0814 \\
\hline MHPRVG & -0.3095 & 0.0834 \\
\hline GM & -0.3131 & 0.0865 \\
\hline HM & -0.3154 & 0.0304 \\
\hline variation & 42.21 & 16.61 \\
\hline & & \\
\hline & & \\
\hline & & \\
\hline & & \\
\hline & & \\
\hline & & \\
\hline & & 0.395 \\
\hline
\end{tabular}


Fig.1 Biplot analysis of adaptability measures for barley genotypes (2018-19)

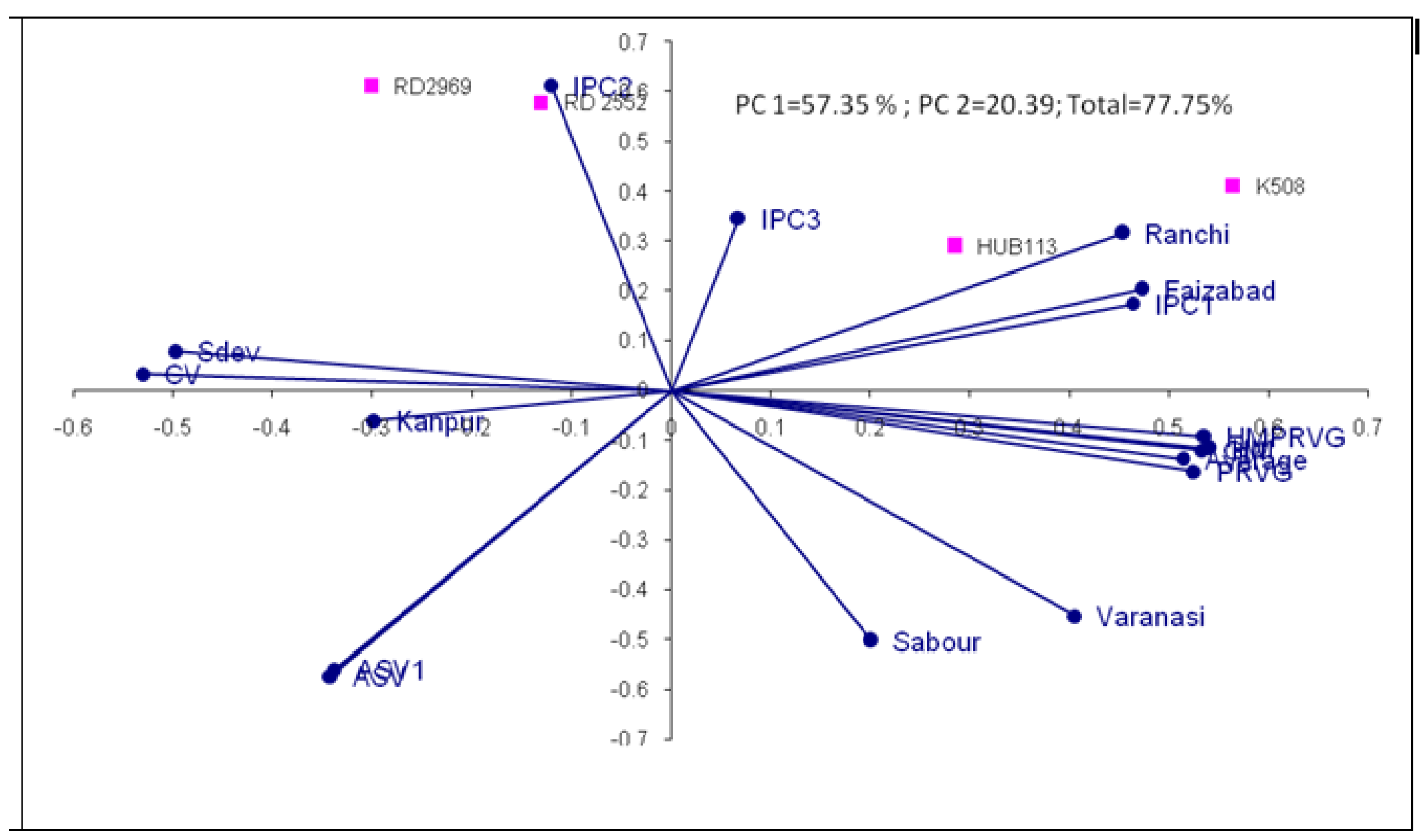




\section{Int.J.Curr.Microbiol.App.Sci (2021) 10(05): 258-271}

Fig.2 Biplot analysis of adaptability measures for barley genotypes (2019-20)

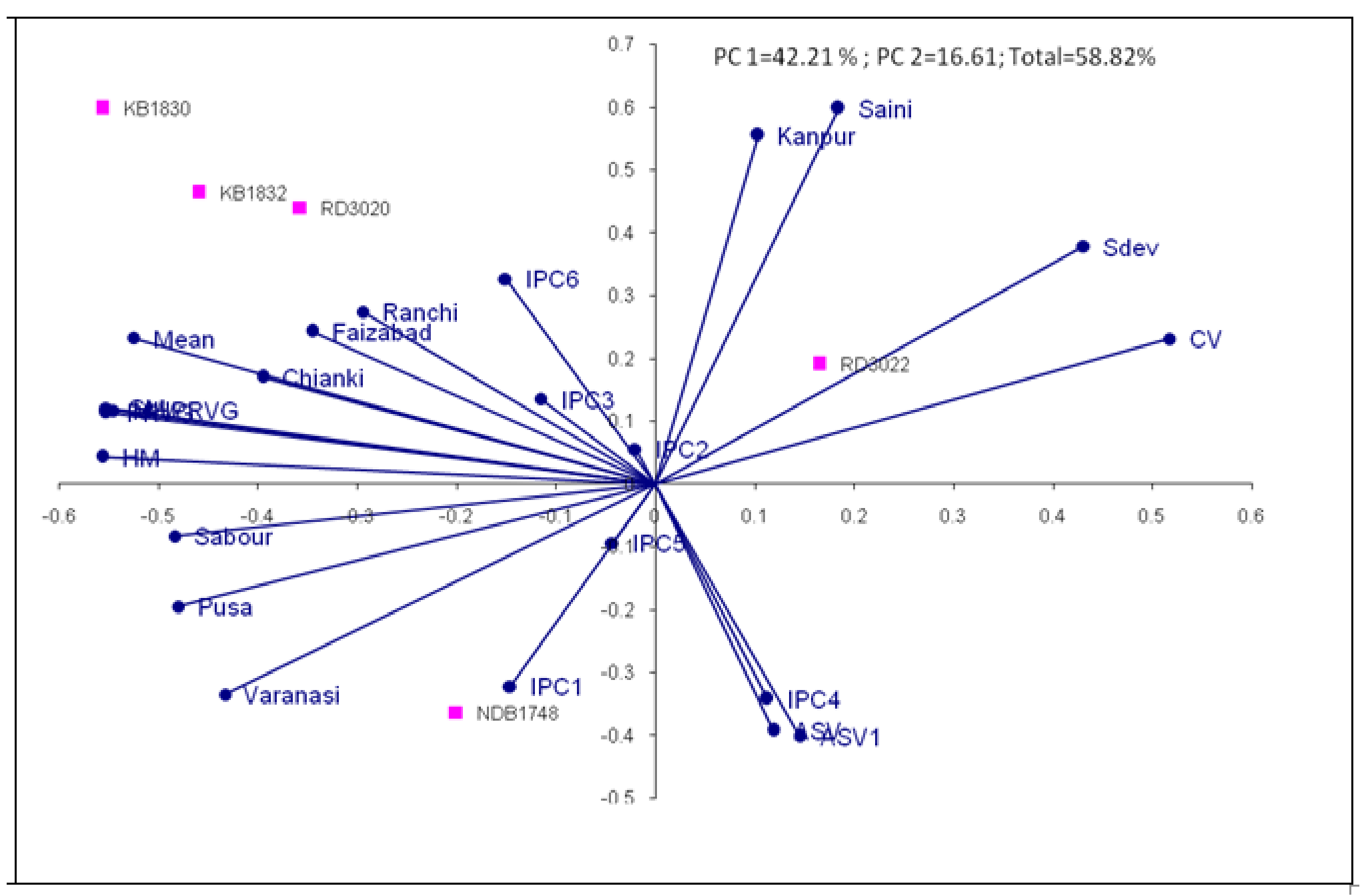


Values of ASV1 selected RD2969, K508 and ASV identified K508, HUB113 barley genotypes (Ajay et al., 2019). Harmonic Mean of Relative Performance of Genotypic Values (HMRPGV) method, the genotypes can be simultaneously sorted by genotypic values (yield) and stability using the harmonic means of the yield so that the smaller the standard deviation of genotypic performance among the locations.

Values of HMRPGV ranked DWRB137, HUB113as the performance of the genotypes among the locations. When considering the yield and adaptability simultaneously, the recommended approach is the relative performance of genetic values (RPGV) over crop years. Relative Performance of Genotypic Values had settled for DWRB137, HUB113 genotypes.

\section{Second year 2019-20}

KB1815, DWRB213, RD3021 were the top ranked genotype as per absolute IPCA-1 scores (Table 8). While for IPCA-2 identified RD3019, NDB1748, KB1815 genotypes would be of choice. Values of IPCA-3 favoredKB1830, PL918, PL925 barley genotypes. As per IPCA-4, KB1815, HUB270, RD3020 genotypes would be of stable performance. IPCA-5 settled for NDB1748, Lakhan, KB1832 barley genotypes. PL918, KB1815, NDB1748 as per IPCA-6 measure. Two significant IPCAs of the AMMI analysis considered by ASV and ASV1 for adaptability behaviour. Values of ASV1 selected KB1815, DWRB213, RD3021 and ASV identified KB1815, RD3021, DWRB213 barley genotypes. Harmonic Mean of Relative Performance of Genotypic Values (HMRPGV) values ranked DWRB213, Lakhan, KB1832 as of stable performance among the locations. Relative Performance of Genotypic Values (RPGV) had settled for Lakhan, DWRB213, KB1832 genotypes.

\section{Biplot analysis}

\section{First year of study 2018-19}

Biplot analysis based on first two highly significant Interaction Principal Components exploited to understand the association if any among adaptability measures.

First two significant interaction principal components contribute to the tune of $57.3 \& 20.4$ to the total for $77.8 \%$ of total GxE interaction sum of squares (Figure 1). Loadings of adaptability measures based on two interaction principal components had mentioned in table 6. CV clustered with Sdev and IPC2 measure in one quadrant and adaptability measures PRVG, HMPRVG along with mean, GM, HM grouped in nearby cluster. Measure's IPC1, and IPC3 joined hands to form another cluster. Clustering of analytic measures ASV and ASV1 observed where close proximity of average yield among adaptability measures of genotypes was of more concern.

\section{Second year 2019-20}

Total contributions to the tune of $58.8 \%$ with respective \% share of $42.2 \& 16.6$ towards the total GxE interaction sum of squares accounted by first two significant interaction principal components (Figure 2). Loadings of adaptability measures based on two interaction principal components had mentioned in table 10. Measure CV clustered with Sdev one quadrant while ASV, ASV1 and IPC4 were grouped together. Measures IPC2, IPC3 and IPC6 clustered with adaptability measures PRVG, HMPRVG, mean, GM, HM in nearby quadrant. Measure's IPC1, IPC5 were observed as outliers in different quadrant. Clustering of analytic measures expressed close proximity among themselves; this implies mean yield of genotypes would be suitable to express adaptability of genotypes 
as far this zone is concerned. High production potential of promising genotypes that respond to favourable environments emphasized by researchers around the country. The presence of complex type $\mathrm{GxE}$ interaction leads to uncertainty in the identification process. More importance had laid to recent techniques to understand the adaptability and stability more precisely. Selection of barley genotypes by the harmonic mean of genotypic values allow the breeders of various crop species around the world to identify the stable and productive genotypes.

\section{Acknowledgements}

Barley genotypes were evaluated at coordinated centers of AICW\&BIP across the country. Authors sincerely acknowledge the hard work of all the staff for field evaluation and data recording.

\section{References}

Agahi K., Jafar Ahmadi, Hassan Amiri Oghan, Mohammad Hossein Fotokian and Sedigheh Fabriki Orang (2020) Analysis of genotype $x$ environment interaction for seed yield in spring oilseed rape using the AMMI model. Crop Breeding and Applied Biotechnology 20(1): e26502012

Ajay B C, Aravind J, Fiyaz R Abdul, Kumar Narendra, Lal Chuni, Gangadhar K, Kona Praveen, Dagla M C and Bera S $\mathrm{K}$ (2019) Rectification of modified AMMI stability value (MASV). Indian J Genet. 79(4): 726-731.

Bocianowsk J, Warzecha T, Nowosad K, \& Bathelt R (2019) Genotype by environment interaction using AMMI model and estimation of additive and epistasis gene effects for 1000-kernel weight in spring barley (Hordeum vulgare L.). Journal of Applied Genetics, 60: 127-135.
Gauch H G (2013) A Simple Protocol for AMMI Analysis of Yield Trials. Crop Science 53:1860-1869.

Hongyu K, García-Peña M, de Araújo L B and dos Santos Dias CT (2014) Statistical analysis of yield trials by AMMI analysis of genotypex environment interaction. Biometrical letters 51:89102.

Kamila N, Alina L, Wiesława P, \& Jan B (2016). Genotype by environment interaction for seed yield in rapeseed (Brassica napus L.) using additive main effects and multiplicative interaction model. Euphytica 208:187194.

Karkee A, Ghimire K H and Joshi, B K. 2020. Evaluation on naked barley landraces foragro-morphological traits. Journal of Nepal Agricultural Research Council. 6:34-43.

Kendel M, Dhami N B and Shrestha J. 2019. Performance evaluation of barley (Hordeum vulgare L.) genotypes in Dolakha, Nepal: from yielding perspective. Journal of Agriculture and Natural Resources 2(1):322-337.

Kharub Ajit Singh, Kumar D., Kumar Vishnu, Malik Rekha, Verma Ramesh Pal Singh, Sharma Indu. (2017). Barley research in India: challenges and opportunities. Haryana, India: Indian Council of Agricultural Research, Indian Institute of Wheat and Barley Research (ICAR-IIWBR).

Kendal E, Tekdal S(2016) Application of AMMI Model for Evolution Spring Barley Genotypes in MultiEnvironment Trials. Bangladesh J. Bot. 45(3): 613-620.

Mohammadi R and Amri A(2008) Comparison of parametric and nonparametric methods for selecting stable and adapted durum wheat genotypes in variable environments. Euphytica 159: 419-432. 
Oyekunle, M., A. Menkir, H. Mani, G. Olaoye, I. S. Usman, S.G. Ado et al., 2017. Stability analysis of maize cultivars adapted to tropical environments using AMMI analysis. Cereal Res. Commun. 45:336-345.

Purchase J L (1997) Parametric analysis to describe $\mathrm{G} \times \mathrm{E}$ interaction and yield stability in winter wheat. Ph.D. thesis. Dep. of Agronomy, Faculty of Agriculture, Univ. of the Orange Free State, Bloemfontein, South Africa.

Rao A R, Prabhakaran VT(2005) Use of AMMI in simultaneous selection of genotypes for yield and stability. Journal of the Indian Society of
Agricultural Statistics 59:76-82.

Resende M D V de and Duarte J B. 2007. Precision and quality control in variety trials. Pesquisa Agropecuaria Tropical. 37(3): 182-194.

Tekdal S, Kendal E (2018) AMMI Model to Assess Durum Wheat Genotypes in Multi-Environment Trials. J Agr Sci Tech. 20: 153-166

Zali H, Farshadfar E, Sabaghpour S H, Karimizadeh R (2012) Evaluation of genotype $\times$ environment interaction in chickpea using measures of stability from AMMI model. Annals of Biological Research 3(7): 3126-3136.

\section{How to cite this article:}

Ajay Verma, R. P. S. Verma, J. Singh, L. Kumar and Singh, G. P. 2021. Feed Barley Genotypes Evaluated for Adaptability under Multi Environment Field Trials of North Eastern Plains Zone of the Country. Int.J.Curr.Microbiol.App.Sci. 10(05): 258-271. doi: https://doi.org/10.20546/ijcmas.2021.1005.033 